\title{
ON THE FADING OF AGB REMNANTS
}

\author{
T. BLÖCKER AND D. SCHÖNBERNER \\ Institut für Theoretische Physik und Sternwarte, Kiel, FRG
}

July 8,1992

We investigated the question how the evolution of post-AGB models depends on their history, i.e. on their initial mass and AGB evolution. Therefore, we calculated the evolution of a 3 and $5 M_{\odot}$ star from the main sequence towards the stage of white dwarfs. These models suffered from 9 and 17 thermal pulses on the AGB, resp., and the common mass-loss law led to final masses of 0.61 and $0.84 M_{\odot}$, resp., which are consistent with reasonable initial-final mass relationships. It was found that more massive AGB remnants fade much more slower than hitherto assumed. Thus, we conclude that only a reliable combination of initial and final mass yields the right fading time scales for more massive post-AGB models. To prove that we have recalculated the evolution of the $3 M_{\odot}$ model with another mass-loss law leading to 86 thermal pulses and a remnant mass of $0.84 M_{\odot}$, a combination which, however, does not comply with initial-final mass relations. Comparing now the post-AGB evolution of the two massive models of equal remnant mass $\left(0.84 M_{\odot}\right)$ but different initial masses ( 3 and $5 M_{\odot}$, resp.) yields completely different fading time scales. Thus we confirm by direct calculations the suggestion of Blöcker and Schönberner (1990, A\&A 240, L11) that not only the remnant mass but also the initial mass determines the time scales of more massive central stars.

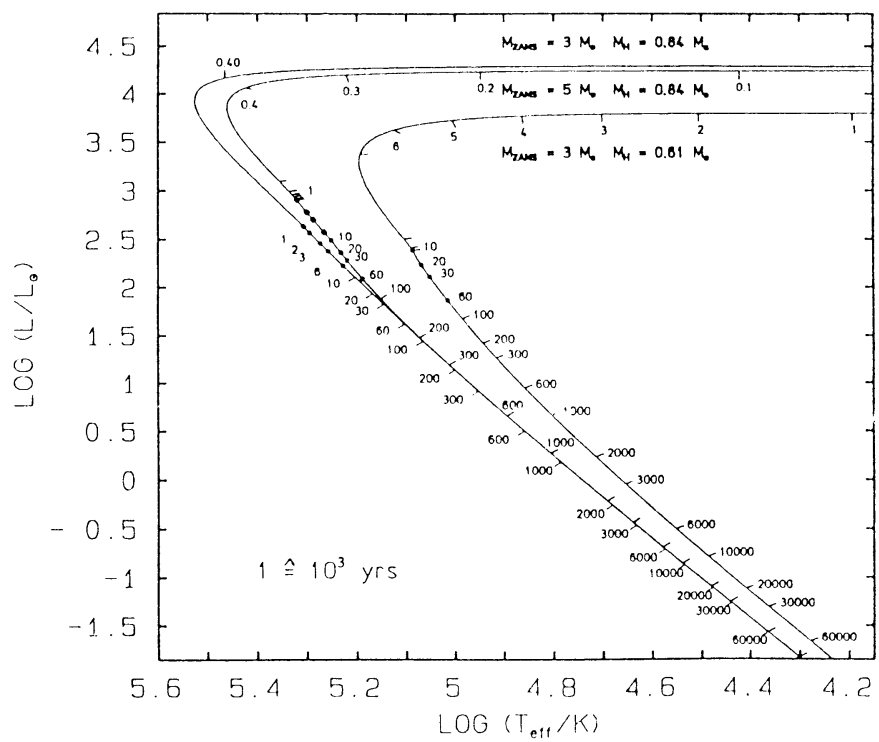

Fig. 1: Evolutionary tracks of three hydrogen-burning post-AGB models (pulse phase $\phi=0.5)$ of $\left(M_{\mathrm{ZAMS}} / M_{\odot}, M_{\mathrm{H}} / M_{\odot}\right)=(3,0.605),(5,0.836)$ and $(3,0.836)$. Note that the latter combination of initial and final mass are not consistent with reasonable initial-final mass relationships. Timemarks are in units of $10^{3} \mathrm{yrs}$. 